\title{
Numerical simulation of the dimensional transformation of atomization in a supersonic aerodynamic atomization dust- removing nozzle based on transonic speed compressible flow
}

\author{
Tian Zhang ${ }^{1,2,3} \cdot$ Deji Jing ${ }^{1,2,3} \cdot$ Shaocheng $\mathrm{Ge}^{4} \cdot$ Jiren Wang ${ }^{1,2,3} \cdot$ Xiangxi Meng ${ }^{1,2,3} \cdot$ \\ ShuaiShuai Ren ${ }^{1,2,3}$
}

Received: 17 May 2019/Revised: 22 January 2020/Accepted: 25 March 2020/Published online: 17 April 2020

(C) The Author(s) 2020

\begin{abstract}
To simulate the transonic atomization jet process in Laval nozzles, to test the law of droplet atomization and distribution, to find a method of supersonic atomization for dust-removing nozzles, and to improve nozzle efficiency, the finite element method has been used in this study based on the COMSOL computational fluid dynamics module. The study results showed that the process cannot be realized alone under the two-dimensional axisymmetric, three-dimensional and three-dimensional symmetric models, but it can be calculated with the transformation dimension method, which uses the parameter equations generated from the two-dimensional axisymmetric flow field data of the three-dimensional model. The visualization of this complex process, which is difficult to measure and analyze experimentally, was realized in this study. The physical process, macro phenomena and particle distribution of supersonic atomization are analyzed in combination with this simulation. The rationality of the simulation was verified by experiments. A new method for the study of the atomization process and the exploration of its mechanism in a compressible transonic speed flow field based on the Laval nozzle has been provided, and a numerical platform for the study of supersonic atomization dust removal has been established.
\end{abstract}

Keywords Aerodynamic atomization · Dust-removing · Laval nozzle $\cdot$ Compressible flow field · Transonic speed · Dimension transform

\section{Introduction}

Based on newly modeled data from the World Health Organization, $92 \%$ of the world population lives in places where air quality levels exceed the WHO ambient air quality guidelines for the annual mean of particulate matter

Deji Jing

jingdeji@lntu.edu.cn

1 College of Safety Science and Engineering, Liaoning Technical University, Fuxin, China

2 Research Institute of Safety Science and Engineering, Liaoning Technical University, Fuxin, China

3 Thermodynamic Disasters and Control of Ministry of Education, Liaoning Technical University, Fuxin, China

4 Safety and Emergency Management Engineering College, Taiyuan University of Technology, Taiyuan, China with a diameter of less than 2.5 micrometers (PM2.5) (World Health Organization 2016). This kind of dust with a very small particle size is called respiratory dust, and it comes from industries such as mining and machining and civilian sources (Betts 2002; Chen et al. 2012; Shi et al. 2009). In addition to endangering human health through inhalation, it can also attach to many toxic and harmful substances, with which it can undergo some complex chemical reactions (Bern et al. 2009; Maertens et al. 2008; Jang and Kamens 1999). Aerodynamic spray is one of the main methods in the field of dust removal, and its efficiency mainly depends on the atomization fineness and particle speed. In the process of collecting fog droplets, the droplet size needs to be close to that of the target dust particles, which results in an extremely high requirement for the atomization efficiency of the nozzle (Dust control theory; Huang et al. 2015; Park et al. 2017; Yang et al. 2018; Jin et al. 2018). 
The Laval nozzle is a kind of device used for fluid control and is widely used in air acceleration. Under certain pressures, the velocity of air in the expanding section breaks the speed of sound (Cengel and Cimbala 2013; Boyanapalli et al. 2013; Kuan and Witt 2013). Because of this characteristic, the structure is often used in jet atomization nozzles (Yang et al. 2003). In this process, the jet fluid instantly breaks down into tiny droplets by overcoming the surface tension in the supersonic flow field due to the relative velocity difference between the flow and the liquid jet (Özkar and Finke 2017; Ghosh and Hunt 2000; Yang et al. 2012). The ultrasonic dusting atomizer is a type of Laval nozzle that is used to produce ultrasonic speeds and break droplets, but this method cannot be considered a good application of the abovementioned method. We must question whether there is a way to avoid the waste resulting from this supersonic process and to find a supersonic atomization dusting nozzle that uses a different process than ultrasonic atomization (Soo 1973). Therefore, to improve the atomization performance of dusting nozzles and explore a kind of ultrafine atomizing nozzle with an ultrahigh efficiency, it is necessary to study the atomization process in Laval nozzles, which is a process in which supersonic gas breaks up droplets; however, no scholars have done this, and the process is difficult to study through experiments. The following analogous studies have been performed.

Several scholars used the gas-liquid two-phase flow method and simulated the atomization process of kerosene jet liquid injection in supersonic transverse laminar flow, but the atomization particle size distribution and gas flow acceleration process were not reflected in the results (Jing and $\mathrm{Xu}$ 2006, Jing 2010; Shunhua and Jialing 2008; Li et al. 2015). Wu (2016) studied the jet atomization process of liquid hydrocarbon fuel in a scramjet combustor by combining experimental research and theoretical analysis. However, no achievements have been made in crushing atomization in the complex transonic process of compressible fluids. Deshpande and Narappanawar (2016) studied the structure and internal flow field characteristics of the Laval nozzle and its related effects; good or bad results were determined only on the basis of air flow parameters such as the velocity of flow, but droplet atomization and the particle size distribution were not considered. Sinha et al. (2015) and Alhussan and Teterev (2017) studied the spray jet process by numerical methods and considered that the nature of instability depends on the density of the jet fluid and the ambient fluid and the velocity of the jet. Inside the Laval nozzle, the air density and temperature are different at different locations, and atomization is a complicated process (Introduction to aerodynamics 2007). However, none of these studies combines the Laval air compression acceleration process with the gas-liquid atomization process.

Supersonic atomization in Laval nozzles is unique, and the density, temperature and speed of the air flow should not be constant. Thus, although it has already performed very well, atomization simulation with transverse laminar flow cannot approximate the real situation inside the atomizer in existing simulations.

In addition, most of the existing Laval studies simulate incompressible airflow, and the dimensions of the simulation model are all two-dimensional. To approximate the real jet atomization process of the transonic compressible flow field, it is necessary to calculate in the 3D model space. This has not yet been achieved by any researchers, mainly because of the following difficulties.

(1) The flow of supersonic gas in the Laval nozzle is a kind of compressible flow, and the density and temperature of the gas are different in different positions.

(2) The flow process in the Laval nozzle is $2 \mathrm{D}$ axisymmetric. In CFD calculations, the 2D axisymmetric model should be applied in the case of COMSOL, which is essentially a $2 \mathrm{D}$ calculation process. In data processing, the dataset of results is rotated with the symmetric axis. This is the correct way to obtain $3 \mathrm{D}$ data. However, the problem is that the results of the droplet atomization model cannot be treated this way because after the rotation, the original data points will develop into rings. Because of this, we cannot observe the distribution of particles in 3D space nor can we obtain the particle size distribution and concentration distribution of a certain interface. Detailed instructions are mentioned later in Chapter 4.

(3) In the $3 \mathrm{D}$ model of COMSOL, it is impossible to calculate the compressible airflow acceleration process in the Laval nozzle because it is unable to be defined axisymmetrically, and the grid cannot be divided axisymmetrically. In addition, it is not accurate to apply symmetric boundaries to the calculation. Detailed instructions are mentioned later.

Therefore, the calculation of the flow field and atomization cannot be realized simultaneously in both $2 \mathrm{D}$ axisymmetric and 3D models. In this way, the characterization of jet atomization in transonic compressible flows in Laval nozzles becomes a seemingly impossible task. However, an approximate method has been found in our study. The rationality of the method is verified by experiments. A new idea for related research and software development will be developed. Although this combination has achieved good results, due to professional limitations, 
the two-way coupling between droplets and compressible flow fields cannot be realized by this simulation, which will need to be solved by software and theoretical researchers. The chapters of the paper are summarized below.

After the introduction, the article contains four chapters. In chapter two, the basic theoretical equations and derivations used in the simulation process are introduced. In the chapter three, the model details and grid division of the numerical simulation, including the grid division and optimization of the 2D axisymmetric model and the grid and grid division of the 3D symmetric model, are introduced. In chapter four, the results and a discussion of the simulation are highlighted. By combining the simulation results of several methods to discuss their infeasibility and reasons, the paper introduces the simulation research method, which realizes the numerical simulation process of droplet atomization in the transonic flow of a compressible gas in 3D space by transforming the dimension. Physical quantitative analysis and atomization experiments are carried out to verify the reliability of the simulation. Finally, the dust reduction test is used to prove the guiding significance of the method for practical application by analyzing the dust removal efficiency. The last section presents the conclusions of the article.

\section{Theory}

\subsection{Theory of transonic compressible flow}

In this work, the simulation is realized by using the finite element method as implemented in CFD of COMSOL Multiphysics software. COMSOL Multiphysics is a special multifield-coupled numerical simulation software, which plays an important role in theoretical research and practical simulation in various fields and can realize the coupling between any multiphysical fields. It is able to establish onedimensional to three-dimensional geometric models and interface with other software. In the mesh division, the direct mesh generator can be used to divide the triangle and tetrahedron units, and some detailed parts can be refined. Local encryption can achieve better results than ANSYS software. In the definition of the variables and equations, embedding can be realized quickly, and the interface is more friendly, for the drawing and other subsequent processing, COMSOL Multiphysics also has a powerful postprocessing function, which can carry out point calculations and sections, draw the parameter evolution graph of the body, and realize the integral operation of the trajectory and variables.

According to the characteristics of the Laval nozzle, some assumptions have been made through the SpalartAllmaras module.
The conservation of mass is a fundamental concept of physics. Within some problem domains, the amount of mass remains constant; mass is neither created nor destroyed. The mass of any object is simply the volume that the object occupies times the density of the object. For a fluid (a liquid or a gas), the density, volume, and shape of the object can all change within the domain with time, and mass can move through the domain (Deshpande and Nitin 2015). The conservation of mass (continuity) tells us that the mass flow rate $m$ through a tube is a constant and equal to the product of the density $\rho$, velocity $\vec{u}$, and flow area $A$ : $m=\rho \vec{u} A$

Considering the mass flow rate equation, we could increase the mass flow rate indefinitely by simply increasing the velocity for a given area and a fixed density. In real fluids, however, because of compressible effects, the density does not remain fixed with increasing velocity. We must account for the change in density to determine the mass flow rate at higher velocities. When the specific heat ratio is $\gamma$, the gas constant is $R_{\mathrm{s}}$, and the temperature is $T$. If we start with using the isentropic effect relations, the equation of state and the mass flow rate equation given above, we can derive a compressible form of the mass flow rate equation based on the definition of the Mach number $M$ and the speed of sound $c$ :

$\vec{u}=M c=M \sqrt{\gamma R_{\mathrm{S}} T}$.

here $M$ is the Mach number.

Substituting Eq. (2) into Eq. (1), when $\rho$ represents the mass density of the fluid, its mass flow rate can be defined by:

$m=\rho A M \sqrt{\gamma R_{\mathrm{S}} T}$.

when $P$ is the pressure, the equation of state is:

$P=\rho R_{\mathrm{S}} T$.

Substituting Eq. (4) into Eq. (3), the mass flow rate is defined by:

$m=A M P \sqrt{\frac{\gamma}{R_{\mathrm{s}} T}}$.

when the total pressure is $P_{\text {tot }}$ and the total temperature is $T_{\text {tot }}$, from the Isentropic flow equations:

$P=P_{\text {tot }}\left(\frac{T}{T_{\text {tot }}}\right)^{\frac{\gamma}{\gamma-1}}$.

Substituting Eq. (6) into Eq. (5), the mass flow rate can be indicated as:

$m=A P_{\mathrm{tot}} M \sqrt{\frac{\gamma}{\mathrm{R}_{\mathrm{s}} T_{\mathrm{tot}}}}\left(\frac{T}{T_{\mathrm{tot}}}\right)^{\frac{\gamma+1}{2(\gamma-1)}}$. 
Another isentropic relation gives:

$\frac{T}{T_{\text {tot }}}=\left[1+0.5(\gamma-1) M^{2}\right]^{-1}$.

Substituting Eq. (8) into Eq. (7):

$m=A P_{\text {tot }} M \sqrt{\frac{\gamma}{\mathrm{R}_{\mathrm{s}} T_{\mathrm{tot}}}}\left[1+0.5(\gamma-1) M^{2}\right]^{\frac{2(\gamma-1)}{\gamma+1}}$.

This equation as shown relates the mass flow rate to the flow area $A$, total pressure $P_{\text {tot }}$ and temperature $T_{\text {tot }}$ of the flow, the Mach number $M$, the ratio of specific heats of the gas $\gamma$ (value of 1.4 in the study), and the gas constant $R_{\mathrm{s}}$ (Mass Flow Rate Equation). Appropriate initial variables are also defined by the equations above in the simulations in our work.

The fluid is assumed to be a Newtonian fluid, which satisfies the Navier-Stokes $(\mathrm{N}-\mathrm{S})$ and the continuity equations.

The compressible form of the Navier-Stokes $(\mathrm{N}-\mathrm{S})$ :

$$
\begin{aligned}
& \rho \frac{\partial u}{\partial t}+\rho(\vec{u} \cdot \nabla) \vec{u} \\
& \quad=\vec{F}-\nabla P+\nabla\left[\left(\mu^{\prime}-\frac{2}{3} \mu\right) \nabla \cdot \vec{u}\right]+\mu \nabla \cdot(\nabla \vec{u}) .
\end{aligned}
$$

where $\boldsymbol{F}$ is the external force per unit volume, and $\mu$ is the coefficient of viscosity. For compressible fluids, generally, there are two coefficients $\mu$ and $\mu^{\prime}$, similar to Hook's elastomer. Both measure the magnitude of the internal friction work caused by the expansion or contraction of a fluid.

Except for extreme cases such as with high temperatures and high frequency sound waves, the motion of gases can be approximately assumed to have a $\mu^{\prime}=0$ because $\mu^{\prime} \nabla \cdot \vec{u}$ is much smaller than $P$. This fact has been indicated in the theory of molecular motion but was originally a hypothesis put forward by Stokes.

$\mu$ varies with the temperature, which conforms to Sutherland's law.

$\mu=\mu_{\text {ref }}\left(\frac{T}{T_{\mu, \text { ref }}}\right)^{\frac{3}{2}} \frac{T_{\mu, \text { ref }}+S_{\mu}}{T+S_{\mu}}$.

In this work, $\mu_{\text {ref }}$ is determined by $T_{\mu \text {,ref }}$ (substituted with the static temperature inlet $T_{\text {stat }}$ ), and $S_{\mu}$ usually valued at $111 \mathrm{~K}$.

The density and the velocity of the fluid further satisfy the continuity equation:

$\nabla \cdot(\rho \vec{u})=0$.

In addition, the fluid should be a steady, axisymmetric, isotropic, and adiabatic flow. In other words, $\frac{\partial u}{\partial t}=0$, so Eq. (10) can be simplified as follows: $\rho(\vec{u} \cdot \nabla) \vec{u}=\vec{F}-\nabla P+\frac{1}{3} \mu \nabla \cdot(\nabla \vec{u})$.

To close the equation, some equations need to be introduced as follows:

$\rho c_{\mathrm{v}}(\vec{u} \cdot \nabla) T=K \cdot T-\rho(\nabla \cdot \vec{u}) T$.

Equation (14) indicates energy conservation.

The thermal conductivity of the fluid satisfies Fourier's Law:

$\vec{q}=-K \nabla T$.

$K=\mu \frac{C_{\mathrm{p}}}{P_{\mathrm{r}}}=\mu \frac{\gamma R_{\mathrm{s}}}{(\gamma-1) P_{\mathrm{r}}}$.

The Prandtl number $P_{\mathrm{r}}$ is a dimensionless parameter in fluid mechanics to characterize the relative importance of the momentum exchange and the heat exchange in the fluid flow. Except for the critical point, the $P_{\mathrm{r}}$ of the gas is almost independent of the temperature and pressure, and it takes the value of 0.72 in this study.

The boundary condition for the wall in the simulation can be restricted as follows:

$\vec{u}=0 ;-\vec{n} \cdot \vec{q}=0$.

Equation (17) assumes that there is no slip and no heat exchange on the outside wall of the nozzle (Patel et al. 2016).

Based on the above assumptions, the physical process of the supersonic flow field in a Laval nozzle can be simulated by solving the above equations when the total pressure $P_{\text {tot }}$, the total temperature $T_{\text {tot }}$ and the Mach number $M_{\text {in }}$ at the inlet are given. In this case, the inlet has a total pressure of 6 atm, a total temperature of $293 \mathrm{~K}$, and a Mach number of 0.01. All parameters are listed in Table 1 .

\subsection{Theory of atomization simulation $(\mathbf{K}-\mathbf{H})$}

First, to represent the continuity of the fluid, the jet was regarded as a continuous droplet column of particles released by several calculation time steps in the transient calculation, as first proposed by Reitz. The particles' attributes are as follows: the dynamic viscosity is $\mu_{\mathrm{p}}$, the surface tension is $\sigma_{\mathrm{p}}$, and the density is $\rho_{\mathrm{p}}$. Because the cross-sectional area of the liquid column is the cross-

Table 1 Input parameters (COMSOL software)

\begin{tabular}{ll}
\hline Boundary & Value \\
\hline Inlet $P_{\text {tot }}(\mathrm{atm})$ & 6 \\
Inlet $T_{\text {tot }}(\mathrm{K})$ & 293 \\
Inlet $M_{\text {in }}$ & 0.01 \\
Outlet $P_{\text {out }}(\mathrm{atm})$ & 1 \\
$R_{\mathrm{s}}(\mathrm{J} /(\mathrm{kg} \cdot \mathrm{K}))$ & 287 \\
\hline
\end{tabular}


sectional area of the particle, when the particle has a mass of $m_{\mathrm{p}}$, the initial radius $R$ is controlled by the $m_{\mathrm{p}}$ when the particles' material attribute is fixed:

$\frac{\mathrm{d} m_{\mathrm{p}}}{\mathrm{d} t}=R$

when the rate of the particle velocity change over time is $\frac{\mathrm{d} v}{\mathrm{~d} t}$, according to Newton's law, the force on the particle in the present is:

$F_{\mathrm{t}}=m_{\mathrm{p}} \frac{\mathrm{d} v}{\mathrm{~d} t}$

The particle receives a drag force of air in the flow field. When the velocity of the air flow is $u$, the dynamic viscosity is $\mu$, the density is $\rho$, and the diameter of the particle is $d_{\mathrm{p}}$, the velocity is $v$, as described by Stokes, and the Shear stress is $\tau_{\mathrm{p}}=\frac{\rho_{\mathrm{p}} d_{\mathrm{p}}^{2}}{18 \mu}$; then, the force of the particle can be expressed as:

$F_{\mathrm{D}}=\frac{1}{\tau_{\mathrm{p}}} m_{\mathrm{p}}(u-v)$

when the velocity difference between the air flow and the droplet at their contact surface is $\Delta u=|u-v|$, and because $\rho / \rho_{\mathrm{p}} \ll 1$ by the Kelvin-Helmholtz instability model (K$\mathrm{H})$, the following is known.

When the gas phase Weber number is $W e_{\mathrm{g}}=\frac{\rho \Delta u^{2} r_{\mathrm{p}}}{\sigma_{\mathrm{p}}}$, the liquid phase Webb number is $W e_{1}=\frac{\rho_{\mathrm{p}} \Delta u^{2} r_{\mathrm{p}}}{\sigma_{\mathrm{p}}}$, where the liquid phase Reynolds number is $\operatorname{Re}_{1}=\frac{\rho_{\mathrm{p}} r_{\mathrm{p}} \Delta u}{\mu_{\mathrm{p}}}$, the Ohnesorge number is $Z=\frac{\sqrt{W e_{1}}}{R e_{1}}, T=Z \sqrt{W e_{\mathrm{g}}}$, the wavelength is $\Lambda_{\mathrm{KH}}=\frac{9.02 r_{p}(1+0.45 \sqrt{Z})\left(1+0.4 T^{0.7}\right)}{\left(1+0.865 W e_{\mathrm{g}}^{1.67}\right)^{0.6}}$, and the maximum growth rate is $\Omega_{\mathrm{KH}}=\frac{0.34+0.385 W e_{\mathrm{g}}^{1.5}}{\left(1+1.4 T^{0.6}\right)(1+Z)} \sqrt{\frac{\sigma_{\mathrm{p}}}{\rho_{\mathrm{p}} r_{\mathrm{p}}^{3}}}$.

The radius of the sub-droplets produced when the mother liquid droplets with a radius of $r_{\mathrm{p}}$ are broken is:

$r_{\mathrm{ch}}=\left\{\min \left(\left(\frac{3 \pi r_{\mathrm{p}}^{2} \Delta u}{2 \Omega_{\mathrm{KH}}}\right)^{1 / 3},\left(\frac{3 r_{\mathrm{p}}^{2} \Lambda_{\mathrm{KH}}}{4}\right)^{1 / 3}\right) \quad \begin{array}{l}B_{0} \Lambda_{\mathrm{KH}} \leq r_{\mathrm{p}} \\ B_{0} \Lambda_{\mathrm{KH}}>r_{\mathrm{p}}\end{array}\right\}$.

when $r_{\mathrm{KH}}$ is the $\mathrm{K}-\mathrm{H}$ broken particle radius, the shear force is $\tau_{\mathrm{KH}}=\frac{3.788 B_{\mathrm{KH}}}{\Lambda_{\mathrm{KH}} \Omega_{\mathrm{KH}}}: B_{\mathrm{KH}}$ is an empirical constant $<10$, which represents the atomization time of particles; the smaller the value is, the earlier the breakup time is, and the faster the atomization rate is (Patel et al. 2015). Then, the particle atomization rate is:

$\frac{\partial r_{\mathrm{KH}}}{\partial t}=\frac{r_{\mathrm{ch}}-r_{\mathrm{KH}}}{\tau_{\mathrm{KH}}}$.
Gas-phase properties were derived from compressible flow simulations, and other parameters in the droplet atomization model were from experimental measurement and set in Table 2 .

By determining the rate of mass flow $m_{\mathrm{r}}$ and the initial radius of particle $d_{0}$, the initial velocity of particle $V_{0}$ and the transient step size were determined, which ensured the continuity of the jet and tracking requirements for particles.

\subsection{Introduction of the new method: dimensional transformation}

The velocity $\boldsymbol{U}$ of any point in a $3 \mathrm{D}$ space $(x, y, z)$ can be decomposed into $V_{z}$ and $V_{\mathrm{r}}$ in a $2 \mathrm{D}$ axisymmetric coordinate system. In addition, in a $3 \mathrm{D}$ space it can be decomposed into $U_{x}, U_{y}$ and $U_{z}$. Their relationship is shown in Fig. 1 .

To ensure the directivity of the vector the decomposition can be expressed mathematically:

Table 2 Input parameters (COMSOL software)

\begin{tabular}{ll}
\hline Parameter & Value \\
\hline Jet $m_{\mathrm{r}}(\mathrm{g} / \mathrm{s})$ & 1.11 \\
Jet $V_{0}(\mathrm{~m} / \mathrm{s})$ & 0.88 \\
Jet $d_{0}(\mathrm{~mm})$ & 1.2 \\
$B_{\mathrm{KH}}$ & 5 \\
Particle $\sigma_{\mathrm{p}}(\mathrm{N} / \mathrm{m})$ & 0.0729 \\
Particle $\rho_{\mathrm{p}}\left(\mathrm{Kg} / \mathrm{m}^{3}\right)$ & 1000 \\
Particle $\mu_{\mathrm{p}}(\mathrm{Pa} \mathrm{s})$ & 0.001 \\
Relative tolerance & $1.0 \times 10^{-5}$ \\
Absolute tolerance & $1.0 \times 10^{-6}$ \\
Step $(\mathrm{s})$ & $\left(0,1.36 \times 10^{-3}, 3 \times 10^{-3}\right)$ \\
\hline
\end{tabular}

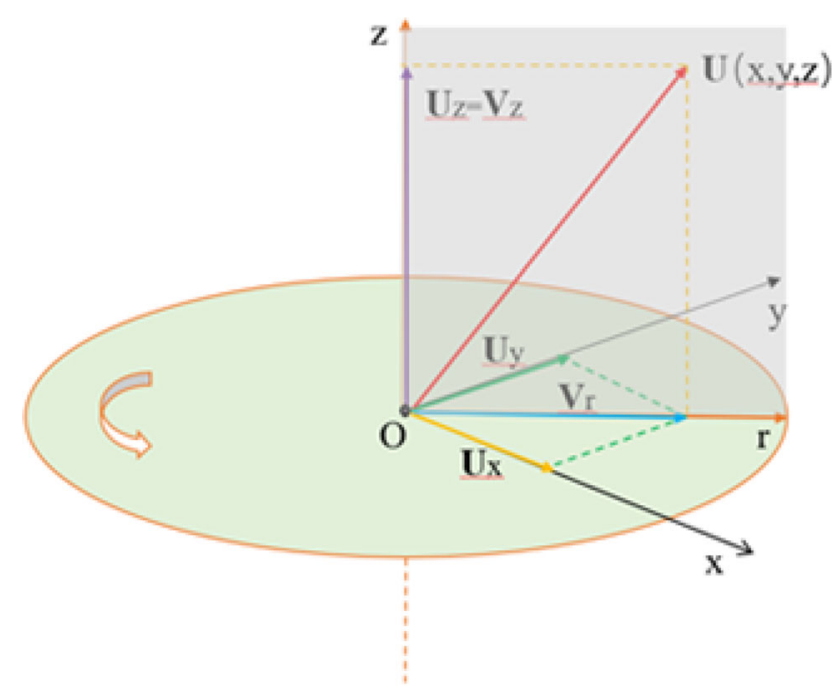

Fig. 1 Vector $\boldsymbol{U}$ of dimension transformation 


$$
\left\{\begin{array}{l}
\vec{U}_{x}=\vec{V}_{r} \cdot x \cdot \sqrt{x^{2}+y^{2}} \\
\vec{U}_{y}=\vec{V}_{r} \cdot y \cdot \sqrt{x^{2}+y^{2}} . \\
\vec{U}_{z}=\vec{V}_{z}
\end{array}\right.
$$

The actual variable setting in the software can be replaced by an interpolation function. The velocity field is set as follows:

$$
\left\{\begin{array}{l}
\vec{U}_{x}=\vec{U}_{x}(x, y, z)=\vec{V}_{r}(x, y, z) \cdot x \cdot \sqrt{x^{2}+y^{2}}, x^{2}+y^{2} \neq 0 \\
\vec{U}_{y}=\vec{U}_{y}(x, y, z)=\vec{V}_{r}(x, y, z) \cdot y \cdot \sqrt{x^{2}+y^{2}}, x^{2}+y^{2} \neq 0 \\
\vec{U}_{x}=\vec{U}_{x}(x, y, z)=\vec{U}_{y}=\vec{U}_{y}(\mathrm{x}, \mathrm{y}, \mathrm{z})=0, x^{2}+y^{2}=0 \\
\vec{U}_{z}=\vec{U}_{z}(\mathrm{x}, \mathrm{y}, \mathrm{z})=\vec{V}_{z}(x, y, z)
\end{array}\right.
$$

\section{Model details and simulation mesh}

\subsection{Model details}

Figure 2 shows a sketch of the simulation setup composed of the Laval Nozzle and the calculation area of the external atmosphere. In this setup, the length of the Laval nozzle and that of the external calculation area were chosen as 30 and $1000 \mathrm{~mm}$. The distances from the throat to the nozzle inlet and outlet are 10 and $20 \mathrm{~mm}$, respectively. The nozzle has an inlet diameter and an outlet diameter of 10.2 and $10 \mathrm{~mm}$ (inlet $>$ outlet), respectively, because of energy conservation and the interior resistance. The atmosphere has a diameter of $1000 \mathrm{~mm}$. The throat has a diameter of $2 \mathrm{~mm}$. All the above parameters were set for the sake of closely approximating reality.

\subsection{Two-dimensional mesh}

The geometry of the nozzle was reproduced with COMSOL Multiphysics software. As described in detail by Moses and Stein (1978), only half of the computational domain was required due to symmetry, as shown in Fig. 3. The mesh was constructed to follow the flow, facilitating convergence of the solution (Singhal and Mallika 2013; Venkatesh and Jayapal 2015; Patel et al. 2016).

In the software, the sidewall curve of the nozzle was drawn in the two-dimensional working plane by the parametric equation. A closed nozzle geometry was generated by drawing perpendicular lines from the two ends of the curves to the center axis, the axis between the two ends and the sidewall curve. Then, a rectangle with a side length of $500 \times 1000 \mathrm{~mm}$ was drawn to indicate the atmospheric area outside the nozzle.

The mesh was divided into triangular meshes because these can capture the variation of the turbulent flow field. The boundary layer and angular refinement used stripshaped quadrilateral meshes, which facilitate spreading along the boundary. The following diagram is a scale graph of the grid; the chromatographic legend on the right represents the mesh cell scale relationship in each subgraph.

An extreme refinement fluid dynamics mesh was used in the nozzle area, a refinement fluid dynamics mesh was used near the nozzle outlet, and a fluid dynamics coarsening mesh was used in the other areas. An additional refinement method based on boundary control was used to refine the throat. All the grid refinement details in the diagram, such as the angle refinement, maximum cell, minimum cell and curvature number, are marked in Fig. 3.

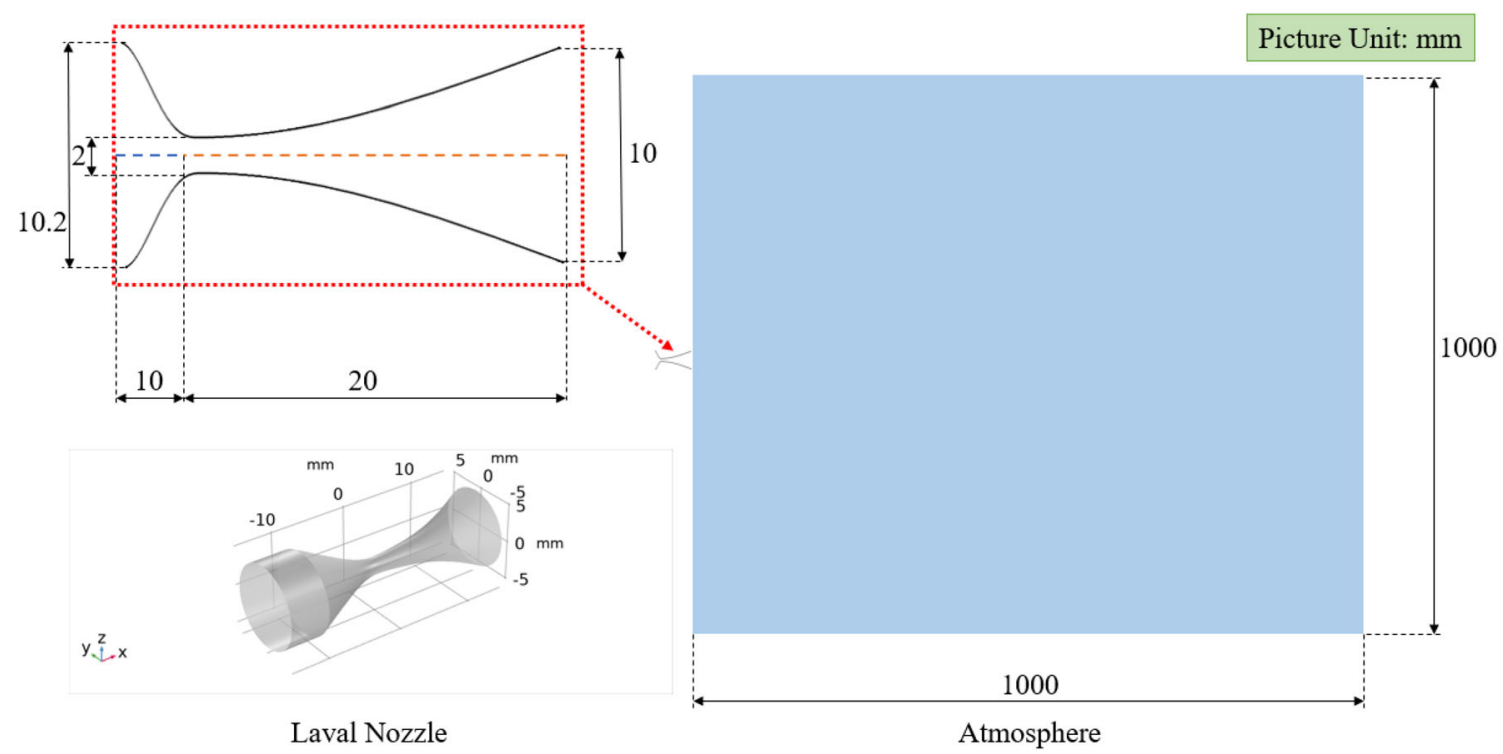

Fig. 2 Model details 


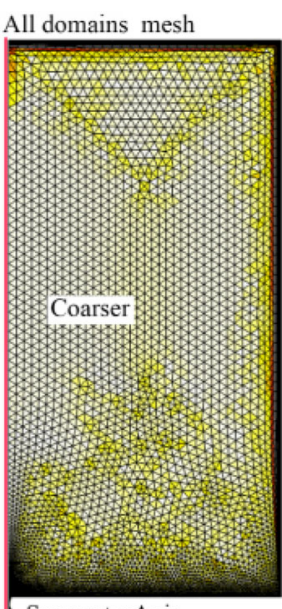

SymmetryAxis

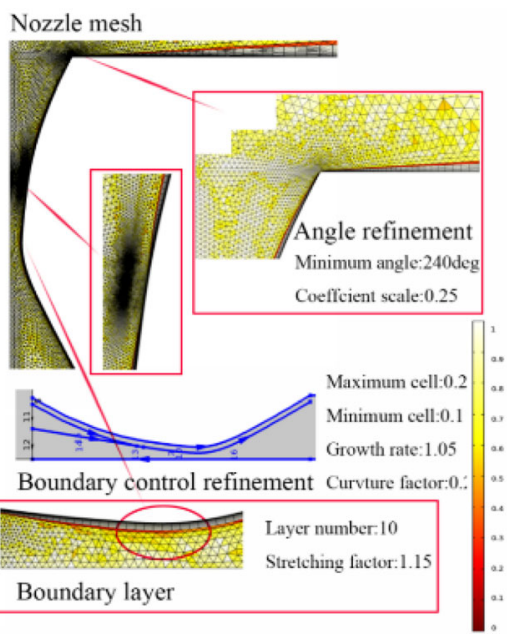

Fig. 3 The 2D mesh and dividing detail

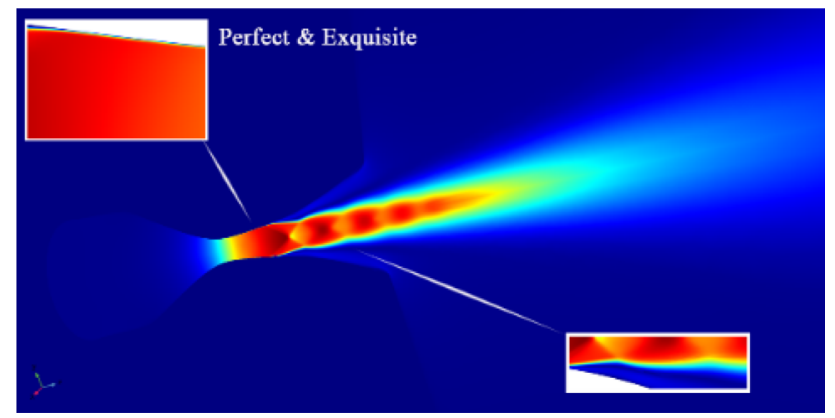

Fig. 4 Simulation result with the optimized mesh

As shown in Fig. 4, the flow field has clear velocity boundaries and was no longer changed by mesh refinement, which ensured that the speed of the microchange could be captured.

\subsection{Three-dimensional mesh}

As shown in Fig. 5, the 3D mesh for the $1 / 4$ symmetric model was divided by a tetrahedral mesh. The entire mesh was divided into four parts to match the calculations. Similarly, the boundary-controlled refinement method was applied, and the specific parameters are shown.

However, in later research, it was proven that the above method was not appropriate. As shown in Fig. 6, a further optimization was made, with the mesh cells presenting an approximate axisymmetric distribution. (a)
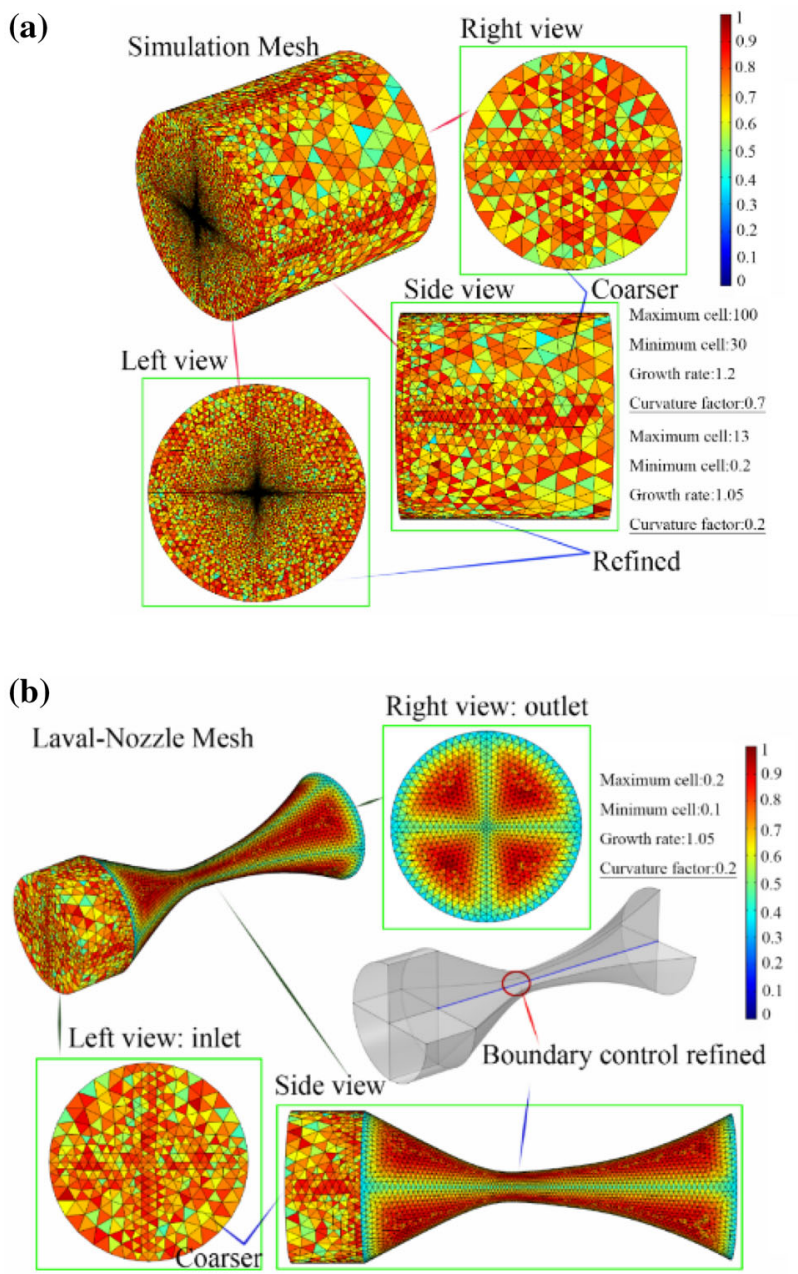

Fig. 5 The 3D 1/4 symmetric mesh and dividing detail, all mesh, nozzle mesh

\section{Results and discussion}

\subsection{Simulation under the $2 \mathrm{D}$ axisymmetric module space}

\subsubsection{Simulation of the 2D flow field}

As shown in Fig. 7, the velocity, pressure, density and dynamic viscosity of flow in the Laval nozzle are different at each position. Accordingly, based on verification using experiments and formulas, the error of the simulation was found to be $3.2 \%$.

\subsubsection{D simulation of droplet atomization}

Under the same model space, the previously calculated flow field results were called. The results of the atomization simulation are shown in Fig. 8. 
(a)

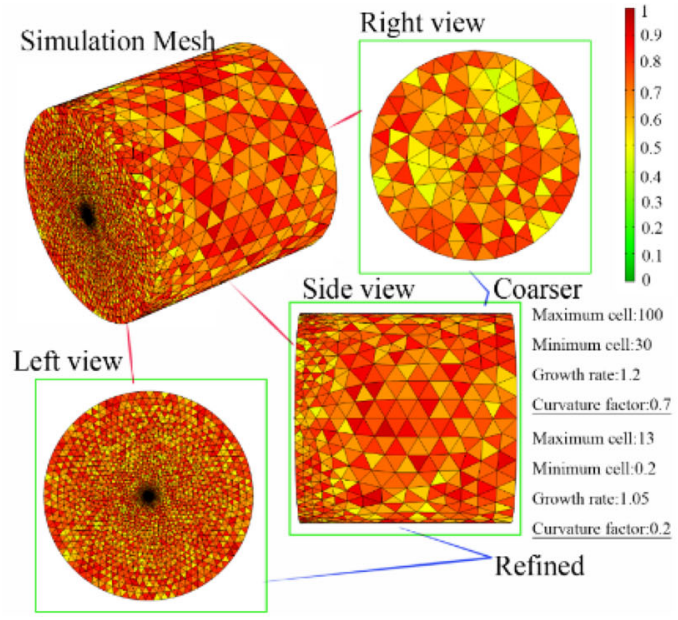

(b)

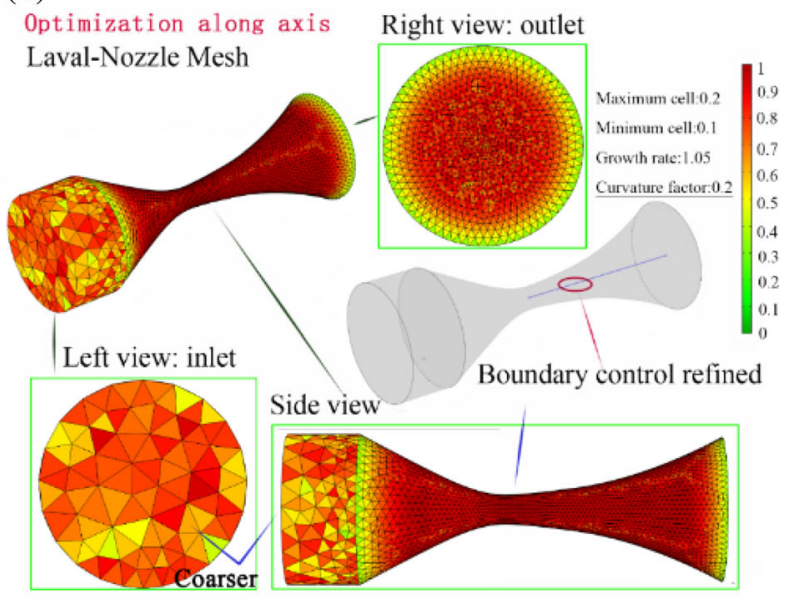

Fig. 6 The 3D nozzle mesh and dividing detail, all mesh, nozzle mesh

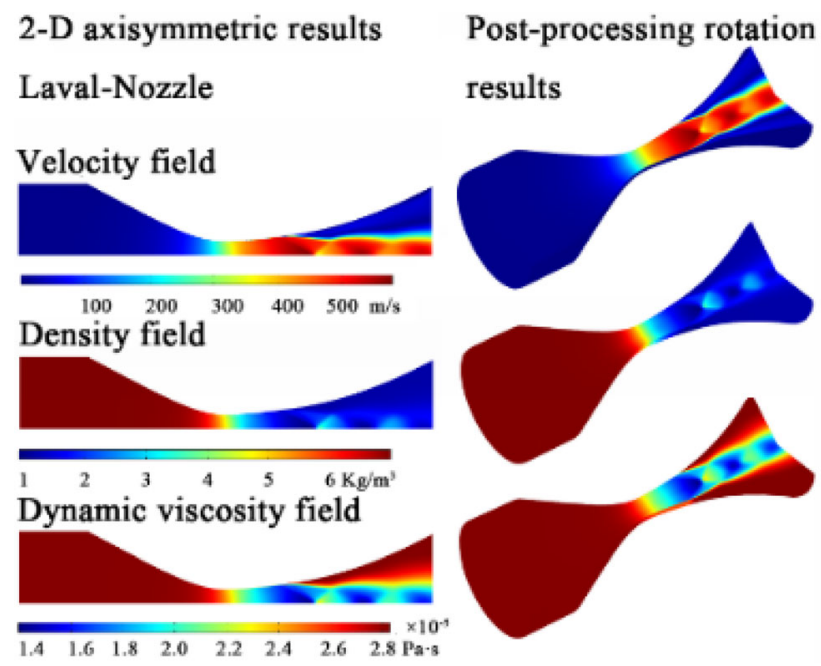

Fig. 7 The 2D simulation results and postprocessing
In Fig. 8, the fog shows a state of a "breathing wave". The particle size near the axis of symmetry is approximately 2 microns, and they accelerate at supersonic speeds in the nozzle. The particle size away from the axis of symmetry is approximately 10 microns, and the particle breaking position away from the axis of symmetry is the subsonic band. Therefore, the spatial distribution of smallsized particles is inside, while that of large-sized particles is outside.

Thus, particles close to the axis of symmetry have higher velocities. In addition, their particle radius is five times that of the particle near the axis of symmetry, and the resistance during the movement is much greater than that of the particle with a smaller radius. This allows particles close to the axis of symmetry to catch up in a very short period with the outer particles that were sprayed earlier, so the wave-like spray distribution is observed macroscopically.

\subsubsection{Problems of the simulation in the $2 D$ axisymmetric module space}

When we want to further discuss the 3D distribution law of particles, we find that the biaxial symmetry model cannot be realized. Just as everyone knows, for a $2 \mathrm{D}$ axisymmetric model, if you want to get a 3D result you need to rotate along the axis, but in Fig. 9, by postprocessing the rotated data set, the results are terrible. Rings were formed by particle rotation, which not only increased the difficulty of analyzing and determining the statistics of the particle distribution but also does not conform to the actual situation. In addition, in this model space, the particles released at the sidewall of nozzle correspond to the water injection mode of the annular discrete slot. Thus, the 3D visualization of particles cannot be realized in a 2D axisymmetric model. Therefore, we want the model space to be threedimensional in the first place.

\subsection{Simulation in the three-dimensional module space}

\subsubsection{Simulation of the flow field}

In Fig. 10, the $1 / 4$ of the flow field was calculated by applying symmetric boundaries in three dimensions, and the other $3 / 4$ was achieved by postprocessing; its mesh is shown in Fig. 5. Although this method produced a correct calculation result on the symmetric boundary, the results between the boundary were wrong. This is because the mesh of the boundary at symmetry cannot approximate axisymmetric conditions.

Thus, the next step is to further approximate the mesh to axisymmetric conditions. 


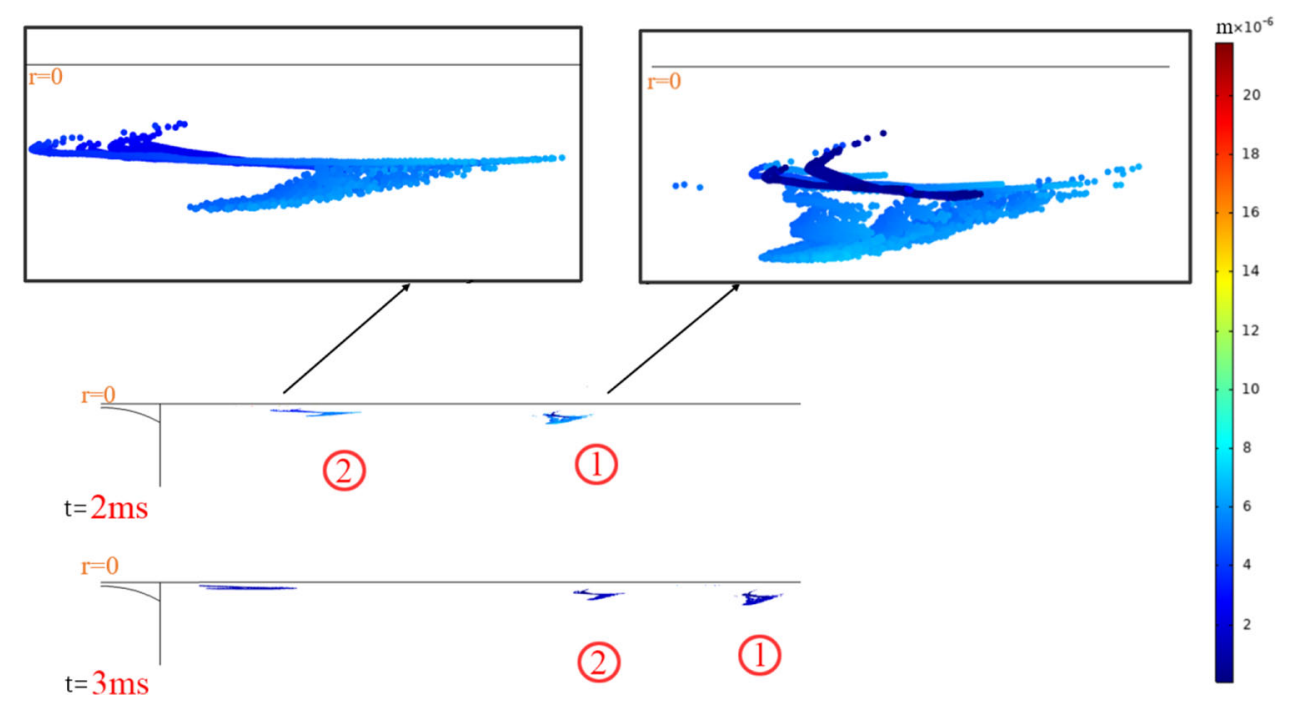

Fig. 8 The 2D atomization simulation results

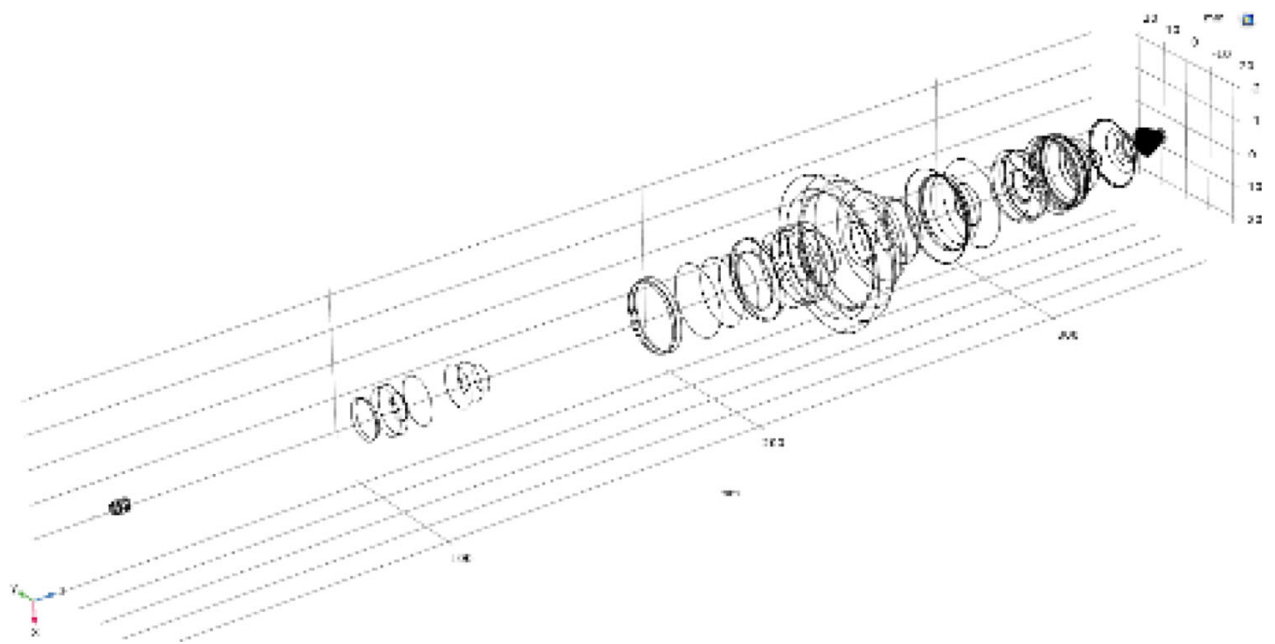

Fig. 9 Rotated particles in a 2D axisymmetric simulation

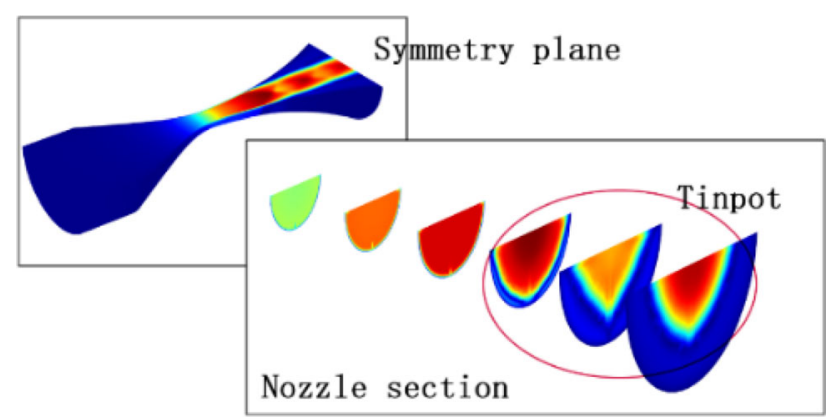

Fig. 10 Simulation results with a symmetric boundary applied

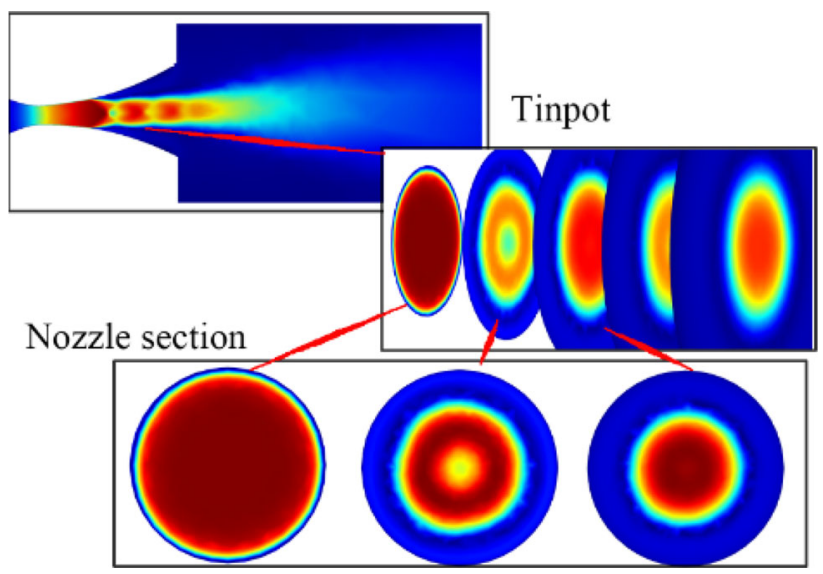

Fig. 11 Simulation results with the mesh optimized along the axis in the 3D model 


\subsubsection{Simulation of the flow field with mesh optimized along the axis in the 3D model space}

In Fig. 11 with the mesh optimized along the axis in the 3D model (mesh in Fig. 6), although the results are better than those obtained by the previous method, some tinpot edges and corners still exist in the flow field, which is not satisfactory.

Because the flow field of the Laval nozzle is totally axisymmetric, the mesh setting cannot be perfect in any case, and it is not appropriate to calculate the 2D axisymmetric problem in a $3 \mathrm{D}$ space.

In a word, it is not accurate to calculate the compressible flow field in the Laval nozzle under the 3D model only, regardless of whether or not it is approximated as symmetric or axisymmetric.

\subsection{Dimension transform numerical simulation of atomization in supersonic atomizing dust- removing nozzle based on transonic speed compressible flow}

Generate the flow distribution data with a 2D axisymmetric spatial basement into 3D basement data.

Import the above data with a 3D basement, including the velocity, density, dynamic viscosity and temperature of the flow field, into the 3D model by means of an interpolating function. In the model settings, some details were set according to the rules of spatial transformation by Eq. (24).

(a)

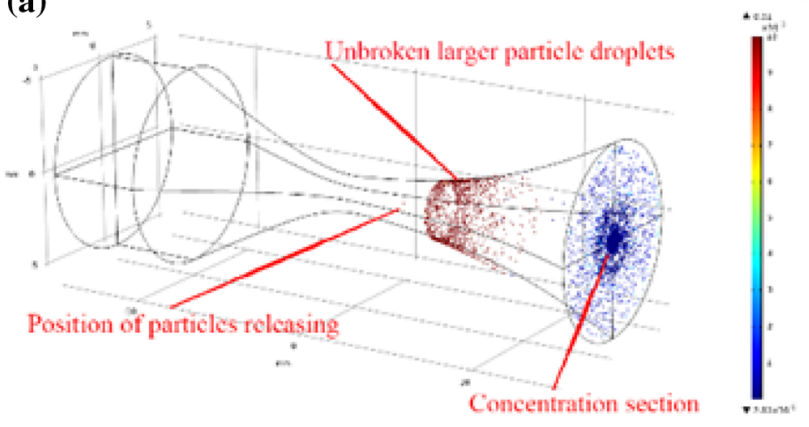

(b)

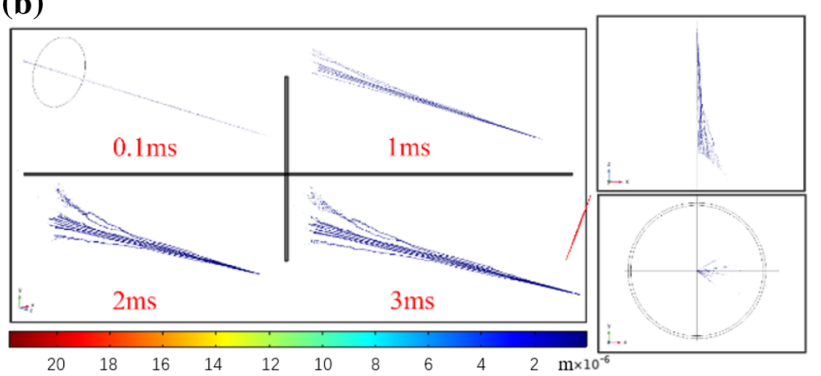

Fig. 12 The simulation results of atomization $\mathbf{a}$ in the nozzle, $\mathbf{b}$ out of the nozzle
In the process of calculating droplet atomization, the above functions were called, and the particle states were calculated by a transient.

The simulation results of particle atomization in the nozzle are shown in Fig. 12.

Calculate the atomization angle and compare it with the experiment result in the next chapter. The position of the released particles is $z=7.5 \mathrm{~mm}$ from the outlet, and the diameter of the particle concentration section is $d \approx 2.5 \mathrm{~mm}$, so $\tan \alpha=d / z=0.356$, and $\alpha=20^{\circ}$.

As shown in Fig. 12, particles with a large size adhere to the wall of the nozzle, and the micron-sized particles are instantly produced and blown out of the nozzle wall at a very high speed, according to the initial velocity direction when the particle is released. The results are basically consistent with the two-dimensional results.

To enhance the effect of the simulation analysis, increase the number of particles released. As shown in Fig. 13, a large number of micron-sized particles present delamination and spray into the outer space of the Laval nozzle.

The phenomenon of a breath wave is obvious. In threedimensional space, the particle coverage cannot reach full

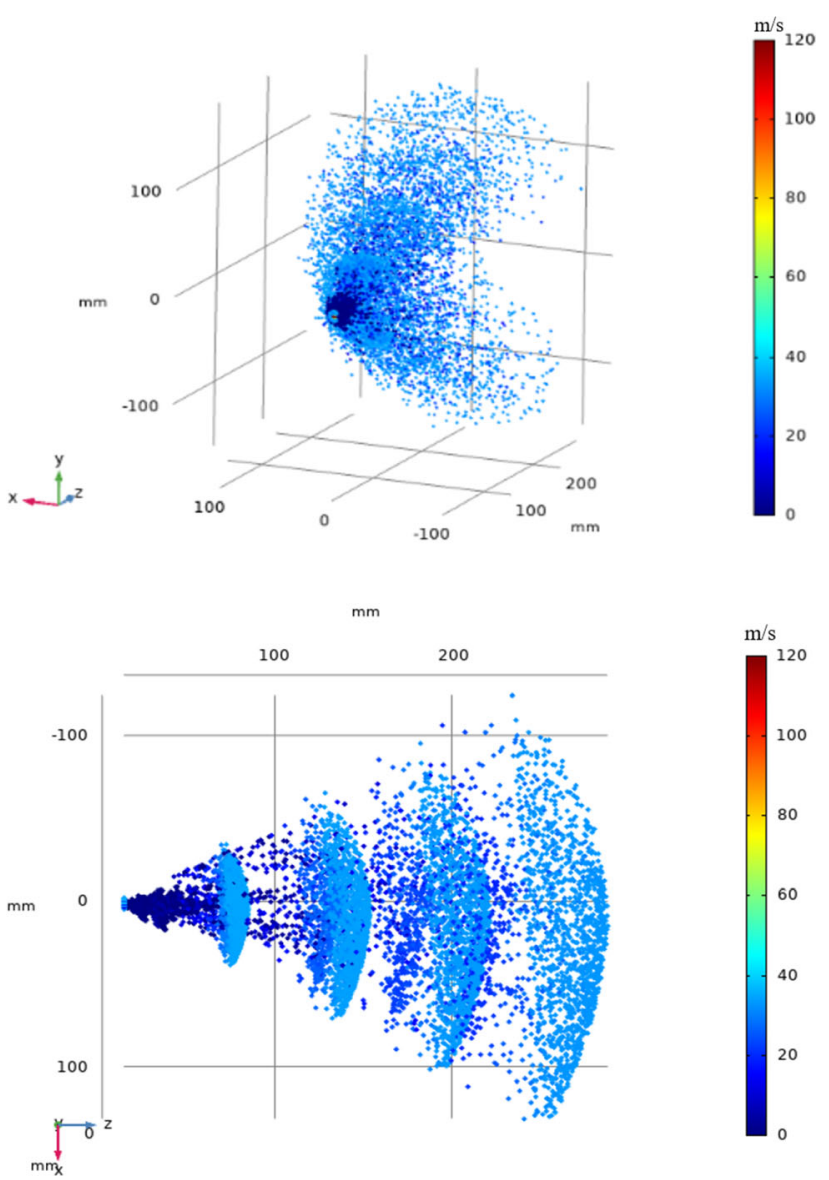

Fig. 13 Atomization results out of the nozzle 
$360^{\circ}$ coverage, which is due to the position of particle release on the sidewall surface that has a certain angle and initial velocity. This phenomenon is called an irregular jet, which is consistent with the results of the subsequent atomization experiment.

As shown in Fig. 14, the particles are released from the sidewall at $0 \mathrm{~s}$ and move slowly. When the flow of particles is in contact with the supersonic band inside the nozzle at 4 $\times 10^{-6} \mathrm{~s}$, the jet droplets are instantly broken into microns and accelerated to subsonic speed (approximately $230-300 \mathrm{~m} / \mathrm{s}$ ) dispersions outside the nozzle.

The closer the axis of symmetry is to the nozzle, the greater the velocity difference between the particles and the flow field is, the more intense the acceleration and breakage are, and the faster the velocity is; however, the latter is ejected out of the nozzle. The axisymmetric stratified distribution of the airflow field causes this phenomenon.

According to the steady state calculation report, the error of the separation group is estimated to be $0.00087,0.00061$; the residual error of the separation group is estimated to be $50,4.9 \times 10^{3}$; the maximum number of iterations of separation 1 (se1) is 1000; the damping factor of the separation step (ss1) is 0.5 ; the damping factor of the turbulent variables is 0.35 ; the tolerance factor is 1.0 ; the solver is PARDISO; the relative tolerance is 0.0012 ; and the transient calculation absolute tolerance is $1.0 \times 10^{-6}$.

\subsection{Rationality analysis by experiment}

Aerodynamic atomization will produce an ultrafine fog curtain and a high-speed wind curtain, fog droplets in the fog curtain will capture the free dust, and the turbulent air
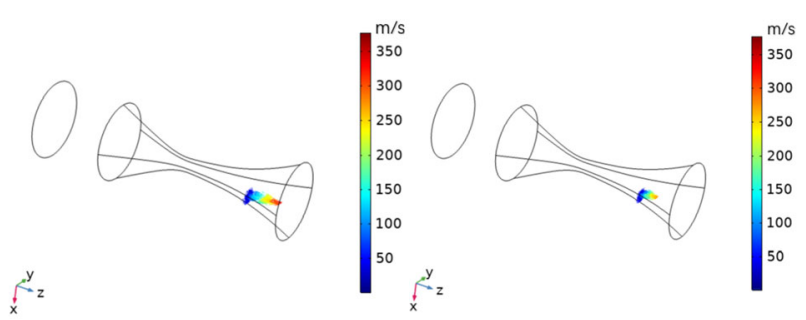

(a) $4 \times 10^{-6}$

(b) $6 \times 10^{-6}$

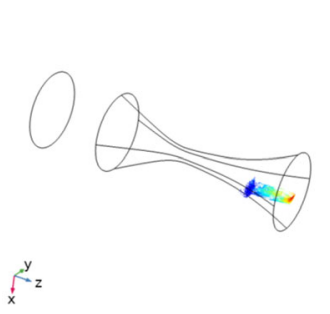

(c) $8 \times 10^{-6}$

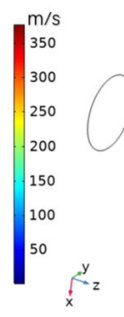

$\mathrm{m} / \mathrm{s}$
350
300
250
200
150
100
50

(d) $10 \times 10^{-6}$

Fig. 14 The supersonic atomization in the nozzle layer formed by the wind curtain will restrain the dust diffusion (Wang et al. 2018; Liu et al. 2019; Cai et al. 2018). Therefore, the accuracy of the simulation is verified by the atomization and dust removal experiments and the guiding significance of the research on the design of dustremoving nozzles.

The experimental parameters were set according to the boundary conditions of the simulation, and the experimental results were compared. The nozzle used in the experiment was designed and manufactured according to the simulation parameters.

In Fig. 15, based on the comparison, the simulated fog droplets were mainly concentrated along the axial direction and had a very high velocity, which was similar to the actual spray situation. The flame shape of the fog and the characteristic of the breath-waves in the process of droplet migration were in good agreement with the experimental results. The main distribution of the particles at the exit position was between 2 and 100 microns, and the spray angle was basically consistent with the experimental results. In both the simulation and the experiment, the water droplets with larger particle sizes were "blown" out along the sidewall of the nozzle outlet.
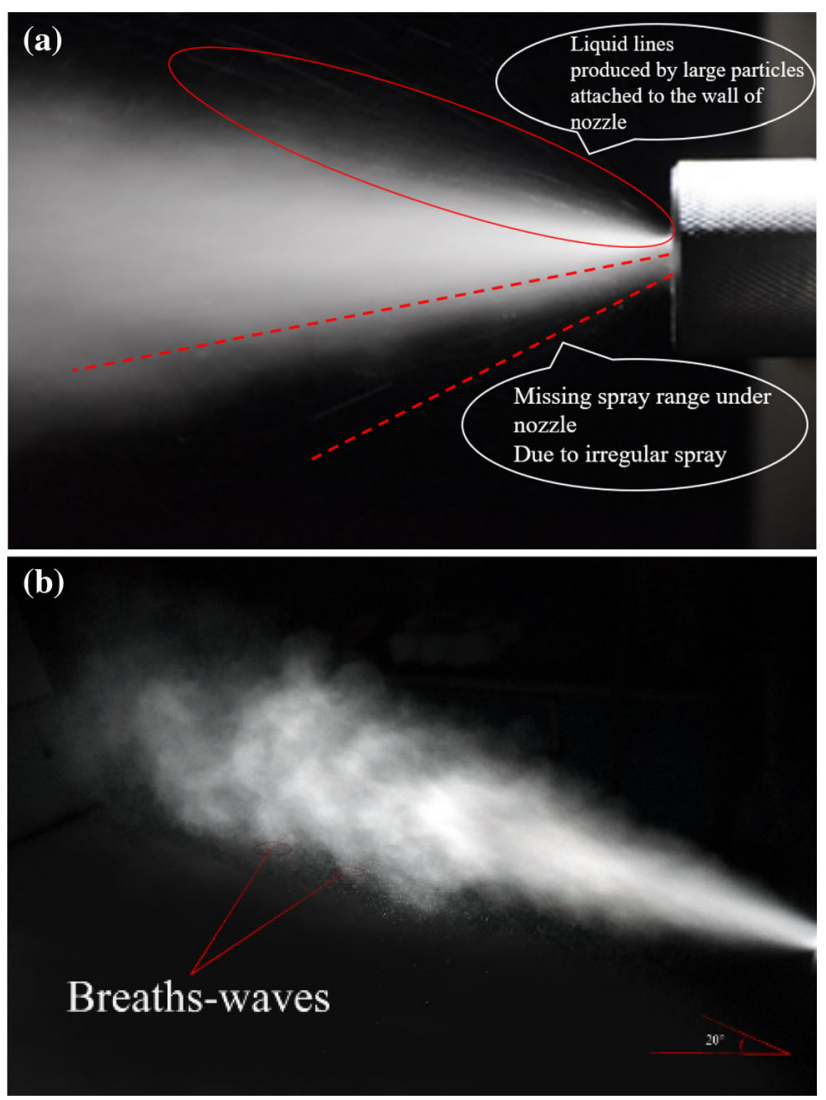

Fig. 15 The comparison of the atomization experiment, a near the nozzle, $\mathbf{b}$ the fog field 


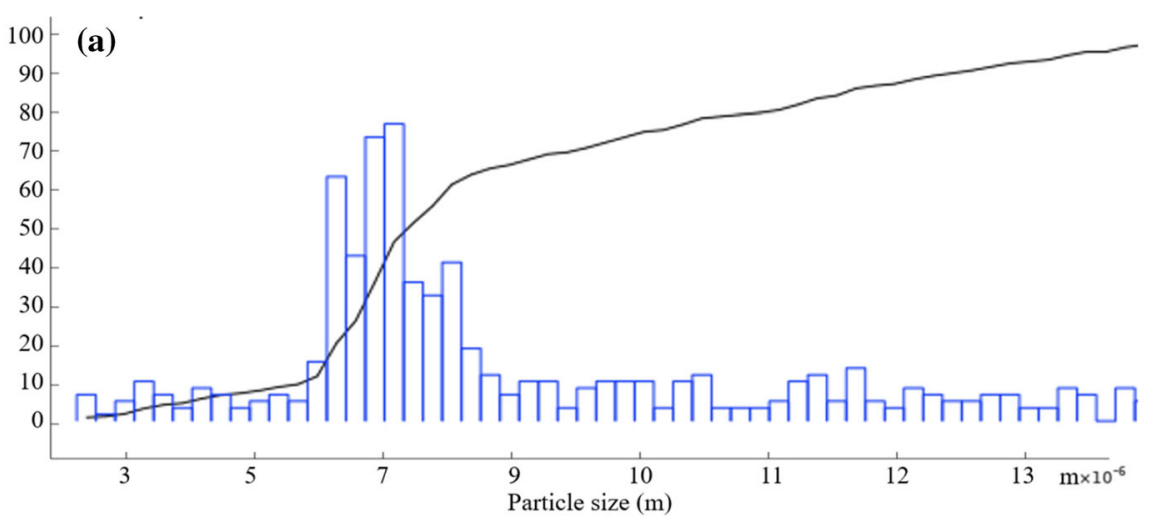

(b)
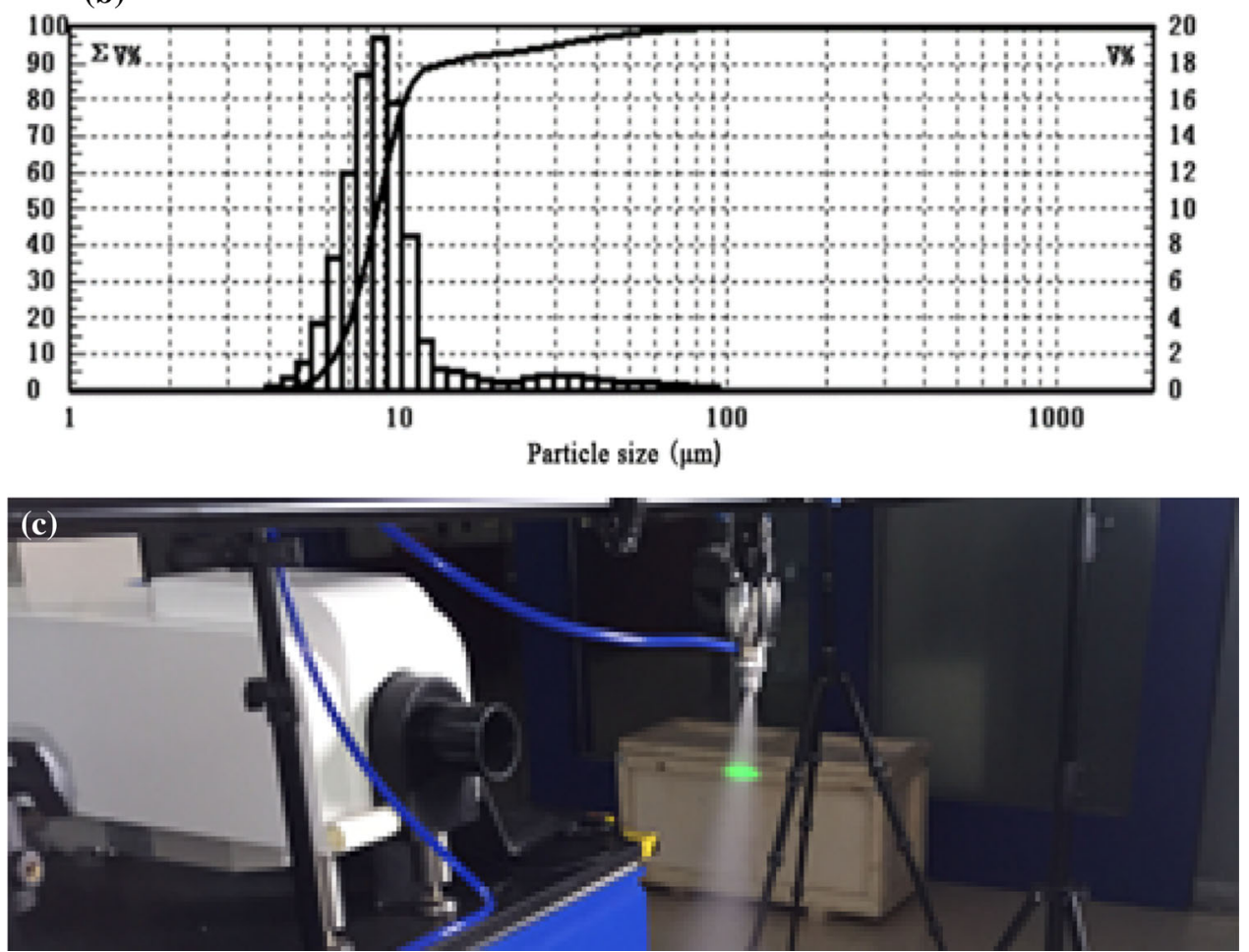

Fig. 16 Atomization experiment to verify a the particle size distribution of the simulation results at $250 \mathrm{~mm}$ from the outlet of the nozzle, $\mathbf{b}$ the particle size distribution of the particle size test by laser diffraction at $250 \mathrm{~mm}$ from the outlet of the nozzle, and $\mathbf{c}$ the particle size test by laser diffraction

In Fig. 16, based on a particle size test by laser diffraction, it is basically proven that the D50 is approximately 8 microns at $250 \mathrm{~mm}$ from the nozzle outlet. This is because the data in the nozzle cannot be measured accurately under the conditions of the existing test instrument, and the accuracy of the simulation can only be verified by the results of the longer distance data outside the nozzle.

The results agree well, which proves that the simulation method is true and reliable.

According to the existing dust removal theory, it is considered that the particle size of droplets used in dust harvesting should be close to that of dust particles (coal dust particles), so for conventional respiratory dust (particle size less than 10 microns) in the coal mining industry, such atomization methods should have a high dust removal efficiency. Therefore, a dust control experiment was carried out to investigate the dust control efficiency of the nozzle on the respiratory coal dust.

In Fig. 17, the experimental process included simulating the air pressure, setting the water flow dust removal experimental conditions, taking dust samples through the dust sampler, weighing the sample before and after drying the mass, calculating the dust concentration, and carrying out the dispersion test. Finally, according to the measured dust concentration results and dispersion test results, the 


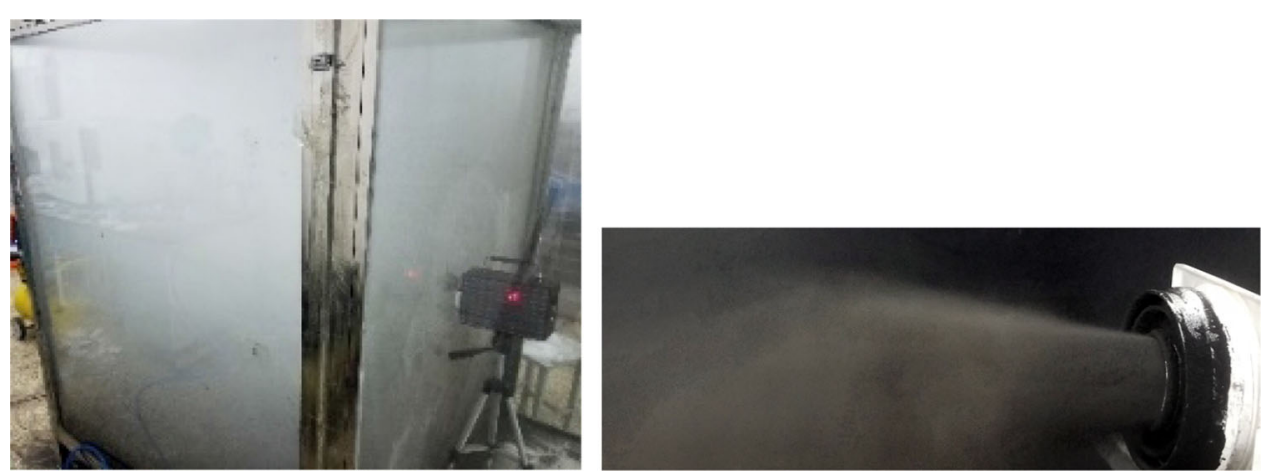

Fig. 17 Dust control experiment (left) dust sampler sampling and atomizing, (right) coal dust emission

transonic atomization dust removal was compared with the conventional respiratory dust removal efficiency.

After the dust removal experiment, this kind of atomization method was shown to achieve a respiratory coal dust removal efficiency of $78.9 \%$ in $1 \mathrm{~min}$, and the capture effect of respiratory dust was much higher than that of the conventional method $(52.03 \%)$, which proves that the simulation method has a basic and guiding significance for the development and research of new technology.

\section{Conclusions}

(1) It is proved that the 2D axisymmetric, 3D symmetry and 3D approximate axisymmetric models cannot accurately simulate the process of droplet fragmentation and atomization in the transonic flow field in three-dimensional space.

(2) Through transforming the dimension, based on the COMSOL software, the droplet crushing and atomization process in the transonic flow field in $3 \mathrm{D}$ space is simulated in the $3 \mathrm{D}$ model, and the atomization experiment is carried out to verify the analysis. The particle distributions of the mesoscopic and macroscopic phenomena are all consistent with a high degree of coincidence.

(3) The physical process, macro phenomena and particle distribution of supersonic atomization are analyzed in combination with this simulation.

(4) Through the atomization and dust removal experiments, the significance of the simulation was proven.

Funding Supported by the National Natural Science Foundation of China (NO: 51704146, 51274116, 51704145).

\section{Compliance with ethical standards}

Conflict of interest We declare that we have no financial and personal relationships with other people or organizations that can inappropriately influence our work, there is no professional or other personal interest of any nature or kind in any product, service and/or company that could be construed as influencing the position presented in, or the review of, the manuscript entitled author.

Open Access This article is licensed under a Creative Commons Attribution 4.0 International License, which permits use, sharing, adaptation, distribution and reproduction in any medium or format, as long as you give appropriate credit to the original author(s) and the source, provide a link to the Creative Commons licence, and indicate if changes were made. The images or other third party material in this article are included in the article's Creative Commons licence, unless indicated otherwise in a credit line to the material. If material is not included in the article's Creative Commons licence and your intended use is not permitted by statutory regulation or exceeds the permitted use, you will need to obtain permission directly from the copyright holder. To view a copy of this licence, visit http://creativecommons. org/licenses/by/4.0/.

\section{References}

Alhussan KA, Teterev AV (2017) AIP conference proceedings [author(s) international conference of numerical analysis and applied mathematics (icnaam 2016) —rhodes, greece (19-25 september 2016)],- -simulation of combustion products flow in the Laval nozzle in the software package SIFIN 1863, 380010

Bern AM, Lowers HA, Meeker GP, Rosati JA (2009) Method development for analysis of urban dust using scanning electron microscopy with energy dispersive X-ray spectrometry to detect the possible presence of world trade center dust constituents. Environ Sci Technol 43(5):1449-1454

Betts KS (2002) WTC dust may cause respiratory problems. Environ Sci Technol 36(13):273A-273A

Boyanapalli R et al (2013) Analysis of composite De-Laval nozzle suitable for rocket applications. Int J Innov Technol Explor Eng $2: 5$

Cai P, Nie W, Chen DW, Yang SB, Liu ZQ (2018) Effect of air flowrate on pollutant dispersion pattern of coal dust particles at fully mechanized mining face based on numerical simulation. Fuel. https://doi.org/10.1016/j.fuel.2018.11.030

Cengel YA, Cimbala JM (2013) Fluid mechanics: fundamentals and applications. Fluid Mech Fundam Appl 6(3):231-259

Chen H, Laskin A, Baltrusaitis J, Gorski CA, Scherer MM, Grassian VH (2012) Coal fly ash as a source of iron in atmospheric dust. Environ Sci Technol 46(4):2112-2120

Deshpande ON, Narappanawar NL (2015) Space advantage provided by De-Laval nozzle and bell nozzle over venturi. Proceedings of 
the world congress on engineering 2015, vol II WCE 2015, July 1-3, 2015, London, UK

Deshpande ON, Narappanawar N (2016) Space optimization through the use of De-Laval nozzle and bell nozzle and its theory. Int Multiconf Eng Comput Sci. https://doi.org/10.1142/ 9789813142725_0034

Dust Control Theory. https://www.wetearth.com.au/dust-controltheory

Ghosh S, Hunt JCR (2000) Spray jets in a cross-flow. J Fluid Mech 365(365):109-136

Huang L, Zhao Y, Li H, Chen Z (2015) Kinetics of heterogeneous reaction of sulfur dioxide on authentic mineral dust: effects of relative humidity and hydrogen peroxide. Environ Sci Technol 49(18):10797-10805

Introduction to aerodynamics (2007) 6-9 Chapter. ISBN: 978-7-56122154-9

Jang M, Kamens RM (1999) A predictive model for adsorptive gas partitioning of SOCs on fine atmospheric inorganic dust particles. Environ Sci Technol 33(11):1825-1831

Jin H, Nie W, Zhang HH, Liu YH, Bao Q, Wang HK (2018) The preparation and characterization of a novel environmentallyfriendly coal dust suppressant. J Appl Polym Sci. https://doi.org/ 10.1002/app.47354

Jing L (2010) Numerical simulation and experimental study of transverse fuel spray in supersonic flow. Doctoral dissertation, Beijing University of Aeronautics and Astronautics

Jing L, Xu X (2006) Numerical simulation of spray in supersonic transverse airflow. Rocket Propuls 32(5):32-36

Kuan BT, Witt PJ (2013) Modelling supersonic quenching of magnesium vapour in a Laval nozzle. Chem Eng Sci 87(2):23-39

Li F, Lu F-G, Luo W, Zhao K, Wang C, Xiong Y (2015) Atomization characteristics of liquid transverse jet in supersonic flow. J Beijing Univ Aeronaut Astronaut 41(12):2356-2362

Liu Q, Nie W, Hua Y, Peng H, Liu C, Wei C (2019) Research on tunnel ventilation systems: dust diffusion and pollution behaviour by air curtains based on CFD technology and field measurement. Build Environ 147:444-460

Mass flow rate equation. www.grc.nasa.gov/WWW/k-12/airplane/ mflchl.html

Maertens RM, Gagné RW, Douglas GR, Zhu J, White PA (2008) Mutagenic and carcinogenic hazards of settled house dust II: salmonella mutagenicity. Environ Sci Technol 42(5):1754-1760

Moses CA, Stein GD (1978) On the growth of steam droplets formed in a laval nozzle using both static pressure and light scattering measurements. J Fluids Eng 100(3):311-322

Özkar S, Finke RG (2017) A classic azo-dye agglomeration system: evidence for slow, continuous nucleation, autocatalytic agglomerative growth, plus the effects of dust removal by microfiltration on the kinetics. J Phys Chem A 121(38):7071-7078
Park J, Ham S, Jang M, Lee J, Kim S, Kim S, Lee K, Park D, Kwon J, Kim H, Kim P, Choi K, Yoon C (2017) Spatial-temporal dispersion of aerosolized nanoparticles during the use of consumer spray products and estimates of inhalation exposure. Environ Sci Technol 51(13):7624-7638

Patel Y, Patel G, Turunen-Saaresti T (2015) Influence of turbulence modelling on non-equilibrium condensing flows in nozzle and turbine cascade. Int J Heat Mass Transf 88:165-180

Patel MS, Mane SD, Raman M (2016) Concepts and CFD analysis of De-Laval nozzle. Int $\mathrm{J}$ Mech Eng Technol (IJMET) 7(5):221-240

Shi Z, Krom MD, Bonneville S, Baker AR, Jickells TD, Benning LG (2009) Formation of iron nanoparticles and increase in iron reactivity in mineral dust during simulated cloud processing. Environ Sci Technol 43(17):6592-6596

Shunhua Y, Jialing L (2008) Numerical simulation of liquid fuel atomization in supersonic flow. Propuls Technol 29(5):519-522

Singhal A, Mallika P (2013) Air flow optimization via a venturi type air restrictor. In: Proceedings of the world congress on engineering, WCE 2013, London, July 3-5, 2013, Vol III

Sinha A, Balasubramanian S, Gopalakrishnan S (2015) A numerical study on dynamics of spray jets. Sadhana 40(3):787-802

Soo SL (1973) Power efficiency for dust collection. Environ Sci Technol 7(1):63-64

Venkatesh V, Jayapal RC (2015) Modelling and simulation of supersonic nozzle using computational fluid dynamics. Int $\mathbf{J}$ Novel Res Interdiscip Stud 2(6):16-27

Wang H, Nie W, Cheng WM, Liu Q, Jin H (2018) Effects of air volume ratio parameters on air curtain dust suppression in a rock tunnel's fully-mechanized working face. Adv Powder Technol 29:230-244

World Health Organization (2016) A global assessment of exposure and burden of disease. Ambient air pollution ISBN: 9789241511353

Wu LY (2016) Breakup and atomization mechanism of liquid transverse jet in supersonic flow. Doctoral dissertation

Yang HT, Viswanathan S, Balachandran W, Ray MB (2003) Modeling and measurement of electrostatic spray behavior in a rectangular throat of pease-anthony venturi scrubber. Environ Sci Technol 37(11):2547-2555

Yang H, Feng LI, Sun B (2012) Trajectory analysis of fuel injection into supersonic cross flow based on schlieren method. Chin J Aeronaut 25(1):42-50

Yang SB, Nie W, Liu ZQ, Peng HT, Cai P (2018) Effects of spraying pressure and installation angle of nozzles on atomization characteristics of external spraying system at a fully-mechanized mining face. Powder Technol. https://doi.org/10.1016/j.powtec. 2018.11.042 